\title{
Medication prescribing and monitoring errors in primary care: a report from the Practice Partner Research Network
}

\author{
A M Wessell, C Litvin, R G Jenkins, P J Nietert, L S Nemeth, S M Ornstein
}

Medical University of South Carolina, Charleston, South Carolina, USA

\section{Correspondence to}

Andrea M Wessell, Department of Family Medicine, Medical University of South Carolina, 295 Calhoun Street, Charleston, SC 29425, USA; Wessell@ musc.edu

Accepted 14 November 2009 Published Online First 22 April 2010

\begin{abstract}
Introduction Medication errors have been associated with poor patient outcomes and pose significant public health consequences. Establishing medication safety quality indicators is crucial to capturing the pervasiveness of preventable errors and is a fundamental first step in the process of improvement. In this article, a study is presented in which a set of medication prescribing and monitoring quality indicators were developed, and adherence to them was assessed among a group of US primary care practices.
\end{abstract}

Methods Twenty Practice Partner Research Network practices in 14 US states with 94 clinicians and 52246 active adult patients participated in the study. All practices use a common electronic medical record with dosing, interaction and monitoring decision support features. A consensus development process was used to select indicators in the categories of inappropriate treatment, dosing, drug-drug and drug-disease interactions, and monitoring of potential adverse events. Data extracted electronically from practices' electronic medical record were used to assess practice-level adherence with the indicator set as of 1 July 2008. Results Thirty medication safety indicators were selected. Across all practices, inappropriate treatment, dosing, drug-drug and drug-disease interactions were avoided in $75 \%, 84 \%, 98 \%$ and $86 \%$ of eligible patients, respectively; monitoring of preventable adverse drug events occurred in $75 \%$ of patients. There was wide variability in practice adherence with the indicators. Discussion The consensus development process was successful in selecting a broad set of primary care medication safety quality indicators. Although aggregate adherence was relatively high in this group of practices, opportunities exist to improve potential errors in treatment selection, dosing and monitoring.

Medication errors have significant public health consequences. Nearly 10 years ago, medication safety garnered significant attention in the USA when the Institute of Medicine called for a focused patient safety effort. ${ }^{12}$ Since then, the majority of improvement efforts have been in the hospital setting, where medications can be tracked and adverse events are apparent. In the ambulatory arena, nearly four billion prescriptions are purchased annually, and the majority of US adults take at least one medication. ${ }^{34}$ Despite this ubiquitous use, surprisingly little is known about the incidence of preventable medication errors in the outpatient primary care setting. Furthermore, methods to assess medication safety are limited, and comprehensive quality indicators are lacking.
The medication use process involves multiple steps, including prescribing, procuring the drug, dispensing, patient adherence and monitoring the patient's response. Errors can occur during any step of the process, making the assessment of medication safety a complex endeavour. Although no standardised taxonomy exists, an adverse drug event $(\mathrm{ADE})$ is generally considered to be any injury due to medication. ${ }^{5}$ More broadly, a medication error is considered to be any error occurring in the medication-use process, regardless of whether injury occurs. ${ }^{6}$ This term encompasses a much more sizeable catalogue of medication-related problems and preventable ADEs, including untreated indications, improper dosing, inappropriate medication use, failing to avoid drug-drug or drug-disease state interactions, failing to monitor a drug's effects and patient nonadherence. ${ }^{7}$ Only a fraction of medication errors result in actual $\mathrm{ADEs}$, yet the avoidance of these errors is essential for optimal patient safety. ${ }^{5}$

The majority of studies regarding outpatient medication safety discuss $\mathrm{ADEs}$ and preventable $\mathrm{ADEs}$, the latter of which are most often associated with errors in the prescribing or monitoring phases. $^{8-12}$ Fewer studies address the broader concept of "medication errors", typically rely on survey or administrative data and are subject to bias or incompleteness. ${ }^{13-15}$ Another limitation to assessing medication safety is the limited number of relevant indicators, most of which focus on pharmacologic care of the elderly. ${ }^{16-18}$ This study addresses the limitations of the current literature by using a consensus development process to select a broad set of primary care prescribing and monitoring indicators and by assessing adherence to them using electronic medical record data.

\section{METHODS}

The study was conducted in the Practice Partner Research Network (PPRNet), a primary care practice-based research network among users of a common electronic medical record (EMR) (McKesson Practice Partner (PP), Seattle, Washington, USA) certified by the Certification Commission for Health Information Technology. Allergy and interaction warnings, dosing calculators and monitoring prompts are decision support tools embedded in PP. PPRNet practices submit quarterly EMR extracts that include patient demographic information such as age, race and sex, as well as clinical information such as diagnoses, medications and laboratory data. An anonymous identifier is assigned to each patient to protect 
confidentiality. Extracted data undergo integrity checks before being aggregated into a longitudinal database.

Twenty 20 PPRNet practices representing 14 US states, 74 physicians, 10 nurse practitioners and 3 physician's assistants volunteered to participate in this study in response to a listserv recruitment message. Practice characteristics are presented in table 1. The Institutional Review Board for Human Research at the Medical University of South Carolina approved the study.

The medication safety indicator consensus development process occurred in five phases. Similar to other published methods, ${ }^{19}$ the phases included preselection, adding and rating, potential indicator review, reflection and combination of similar measures. PPRNet members and participating clinicians served as the expert panel in this process. First, 90 potential medication prescribing and monitoring indicators were identified, drawn from published medication-related quality indicators ${ }^{18} 20-24$ and the functionality embedded in the decision support features of PP. Next, a session was conducted at the 2007 PPRNet network meeting in which 12 primary care physicians provided iterative feedback to the research team on the content of the indicator set and a recommended number of indicators for inclusion in practice reports. Based on an available taxonomy, ${ }^{7}$ indicators were grouped into five categories: potentially inappropriate treatment, inappropriate dosing, drug-drug interactions, drug-disease interactions and monitoring or prevention of ADEs. Third, the lead clinician from each participating practice then completed a survey to prioritise 40 of the 90 proposed indicators. Fourth, indicators with greater than the median number of votes for inclusion (median 9.5, $\mathrm{n}=52$ indicators) were reviewed by the research team for primary care relevance and feasibility of programming from available EMR data. Clinicians from four practices also provided feedback on pilot practice performance reports. Eight indicators were excluded

Table 1 Practice characteristics $(n=20)$

\begin{tabular}{lc}
\hline Practice characteristic & Results \\
\hline Specialty & \\
Family medicine & 17 \\
$\quad$ Internal medicine & 3 \\
Geographic location (n) & \\
South & 9 \\
Midwest & 5 \\
West & 4 \\
Northeast & 2 \\
Urban/rural location* (n) & \\
Urban & 18 \\
Rural & 2 \\
No of clinicians (range 1-36, n) & \\
1 or 2 & 11 \\
3 or 4 & 7 \\
$\geq 10$ & 2 \\
Practice type (n) & \\
Physician owned & 14 \\
Hospital owned & 3 \\
Other (university practice, community-based & 3 \\
primary care, non-profit) \\
Duration of PPRNet membership (range 2-13 years, n) \\
2-5 years \\
$6-10$ years \\
$>10$ years \\
Active adult patients per practice (median, IOR) \\
\hline PPRNt Pratice Patrer Reseach Network. \\
\end{tabular}

PPRNet, Practice Partner Research Network.

*Defined by the practice zip code using the US Department of Health and Human Services Health Resources and Services Administration. Rural-Urban Commuting Area Codes (V.2.0). http://depts.washington.edu/uwruca/ruca-download.php during this step because of limited relevance (eg, monitoring of thyroid-stimulating hormone in patients on lithium) or programming feasibility (eg, change in serum creatinine of less $30 \%$ after ACE inhibitor initiation), resulting in a final set of 44 indicators. Finally, groups of similar measures, such as nonsteroidal anti-inflammatory drug-disease interactions and serum creatinine monitoring, were combined, which further streamlined the set to 30 indicators. Each indicator was supported by at least one of the decision support features.

Data electronically extracted from the EMRs of participating practices were used to assess practice-level adherence by category and for each specific indicator as of 1 July 2008. Active patients were defined as those with a progress note in the prior 12 months who were not subsequently identified as deceased, transferred or inactive. Prescriptions were considered active if they were written in the last year and lacked a discontinuation date. A discontinuation date was calculated for prescriptions with a documented duration. Active problems were obtained from documentation on a problem list or as a diagnosis code within the last 3 years. For renal dosing indicators, creatinine clearance was estimated using the Cockcroft-Gault equation based on the most recent serum creatinine and actual body weight. ${ }^{25}$ Ideal body weight was not calculated due to the absence of consistent recording of height data in the EMR. Practice adherence was calculated as the number of eligible patients who met the indicator criterion divided by the total number of eligible patients as defined in table 2. Median and benchmark adherence across the 20 participating practices were then calculated for each indicator. The PPRNet benchmark uses the Achievable Benchmark of Care algorithm, which assures that high-performing practices with few eligible patients do not overly influence the final benchmark. ${ }^{40} 41$ For each of the indicator categories, summary adherence was calculated as the percentage of patients meeting criteria for every indicator for which they were eligible out of the total number of patients eligible for any indicator within that category. Thus, if patients were eligible for more than one indicator in a given category, they had to meet criteria for each indicator to be classified as adherent.

\section{RESULTS}

As of 1 July 2008, there were 52246 active patients over 18 years of age in the 20 participating practices and 31379 patients $(60.1 \%)$ were eligible for at least one medication safety indicator. Table 2 describes the PPRNet Medication Safety final indicator set, total number of eligible patients by indicator, the practice adherence range, median and benchmark. The number of practices meeting the benchmark is also presented. Practice adherence was highly variable for indicators across categories, with the exception of avoiding potential drug-drug interactions in which medians ranged from $95 \%$ to $100 \%$. High medians were also observed for the drug-disease interaction category. The lowest median practice adherence occurred for dosing, treatment selection and monitoring categories. Across all indicators, there were 120626 opportunities for potential errors to occur, of which errors were avoided $88 \%$ of the time.

Summary adherence by medication safety category is presented in table 3. Overall, the highest adherence was observed for the category of avoiding drug-drug interactions, with potential drug-drug interactions being avoided in $98 \%$ of eligible patients. The lowest adherence rates were for avoidance of inappropriate treatment and appropriate monitoring of potential ADEs, which both occurred in $75 \%$ of eligible patients. 


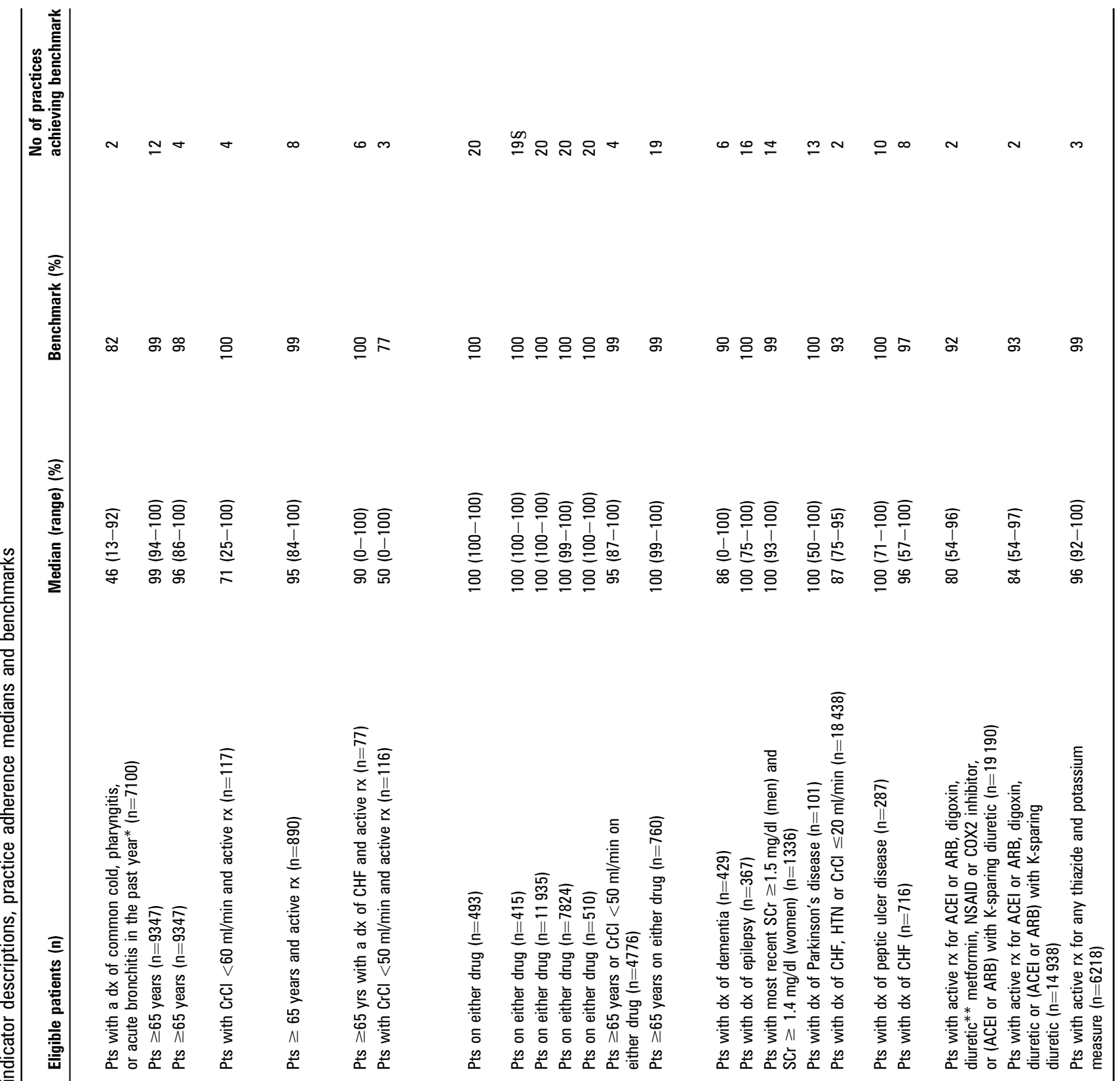




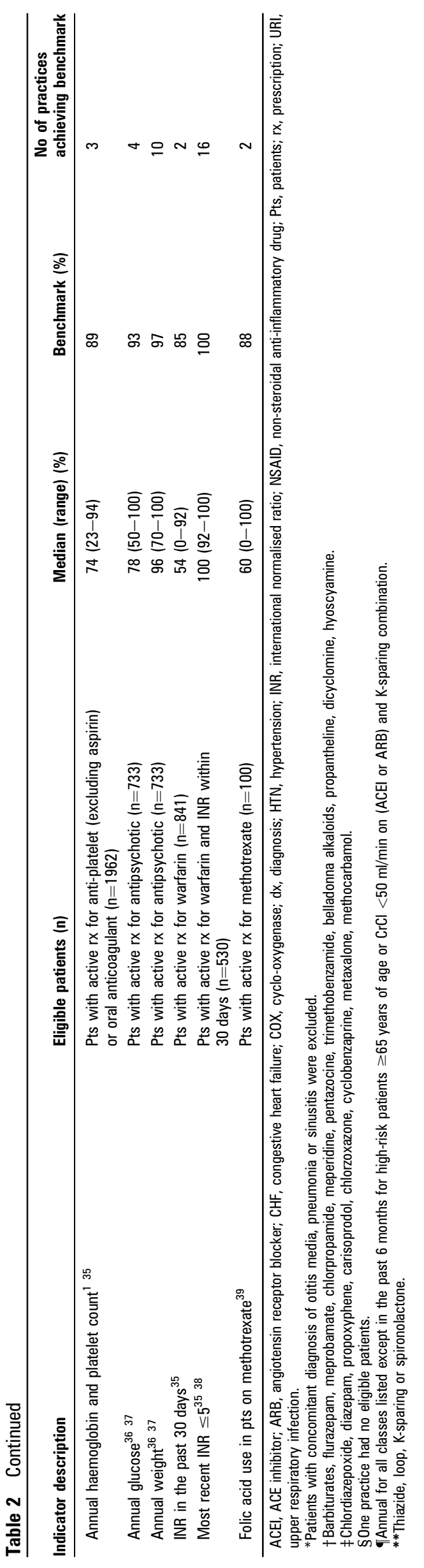

Table 3 Summary adherence across 20 Practice Partner Research Network practices

\begin{tabular}{lll}
\hline Medication safety category & $\begin{array}{l}\text { Total No of } \\
\text { eligible patients }\end{array}$ & $\begin{array}{l}\text { Summary } \\
\text { adherence (\%) }\end{array}$ \\
\hline $\begin{array}{l}\text { Avoiding potentially inappropriate } \\
\text { treatment }\end{array}$ & 15388 & 75.1 \\
$\begin{array}{l}\text { Avoiding potentially inappropriate dosing } \\
\text { Avoiding potential drug-drug interactions }\end{array}$ & 1147 & 84.0 \\
$\begin{array}{l}\text { Avoiding potential drug-disease } \\
\text { interactions }\end{array}$ & 19037 & 98.4 \\
$\begin{array}{l}\text { Monitoring/preventing potential adverse } \\
\text { drug events }\end{array}$ & 20045 & 86.0 \\
\hline
\end{tabular}

\section{DISCUSSION}

In this study, we developed a broad set of prescribing and monitoring quality indicators for use in primary care practice. The selected indicators reflect five key aspects of medication safety, and adherence was assessed from the EMR data of 20 primary care practices. High adherence with indicators such as avoiding inappropriate medications in the elderly, drug-drug interactions and some drug-disease interactions may be attributed to embedded EMR alerts that require a response from clinicians before completing a prescription. Lower adherence with renal dosing and monitoring indicators may be due to the need for further action by the clinician, such as invoking additional clinical decision support or ordering laboratory tests.

Our ability to compare these findings with others is limited due to the relatively sparse existing literature. However, our findings are similar to others for medication monitoring and medication use in the elderly and somewhat better for the specific indicator of anticholinergic medications in patients with dementia. ${ }^{18}$

This study has several limitations. First, despite the breadth of the selected indicators, this set does not represent the full spectrum of medication safety. Categories such as therapeutic duplications, untreated indications, medications without an indication and medication use in pregnancy are not included. ${ }^{7}$ These categories were excluded before indicator development due to lack of defined indicators or decision support tools, or during the development process due to low clinician interest. A formal round of reliability testing, as suggested by some researchers, ${ }^{19}$ was not applied to the consensus development process for this set of indicators based on the level of agreement from participating clinicians during the rating stage. Calculated adherence to study indicators may be biased if EMR medication lists were incomplete, ${ }^{42}$ if problem lists or diagnosis codes were inaccurate or if laboratory data were not recorded in the EMR. The exclusion of deceased patients may have led to an underestimation of errors. Additionally, variability in EMR use across the sample of practices may have influenced the use of decision support features as well as adherence. Findings from this study may not be generalisable to practices without medicationspecific decision support tools or those who do not participate in quality improvement projects.

Despite these limitations, this is, to our knowledge, the first evaluation of multiple categories of medication errors in a geographically diverse group of independent US primary care practices. Since clinicians contributed to their development, these indicators are more likely to be accepted by them. Although EMR data may be incomplete, the calculation of prescribing and monitoring error rates from clinical EMR data overcome the inherent limitations of surveys or administrative data sets. This study provides a replicable set of medication safety indicators and specificity to the scope of prescribing and monitoring improvement needed in primary care practices. 
We view our current study as an initial step in the process of exploring safe prescribing and monitoring in primary care practice. Our preliminary findings are that primary care practices with EMRs have relatively high adherence with a broad set of medication safety indicators but that opportunities exist to improve in the areas of inappropriate treatment, dosing and monitoring. Additional studies should involve larger numbers of practices, assess the relationship between use of specific decision support tools and medication errors, examine the characteristics of practices that achieve high adherence with medication safety indicators and test interventions designed to improve adherence with them.

\section{CONCLUSION}

Due to the expansive array of potential medication errors, developing strategies to identify such errors is a daunting task; however, it is also a crucial step in the pathway to improving medication safety. Furthermore, identifying errors requires the development of a comprehensive set of medication safety indicators. To our knowledge, this project demonstrates the first application of a broad set of prescribing and monitoring quality indicators to a group of primary care practices in the USA. Overall, $88 \%$ of potential medication errors as defined by 30 selected indicators were avoided in participating PPRNet practices. Ample opportunities exist to improve medication safety at the primary care practice level, specifically in the areas of treatment selection, dosing and monitoring.

Acknowledgements This work was presented in part at the 2008 Annual Meeting
of the North American Primary Care Research Group and published in abstract form.

Funding This study was funded by the Agency for Healthcare Research and Quality Ambulatory Safety and Quality Program, Improving Quality through Clinician Use of Health Information Technology Grant No. 1R18HS017037-01. The funding agency had no role in the study design; in collection, analysis and interpretation of data; in writing of the report; and in the decision to submit the paper for publication.

Competing interests AMW and SMO are consultants for McKesson Practice Partner, the vendor of the electronic medical record used by practices in this study.

Ethics approval This study was conducted with the approval of the Institutional Review Board for Human Research at the Medical University of South Carolina.

Provenance and peer review Not commissioned; externally peer reviewed.

\section{REFERENCES}

1. Aspden P, Wolcott J, Bootman JL, et al. Institute of Medicine (US). Committee on Identifying and Preventing Medication Errors. Preventing medication errors. Washington, DC: National Academies Press, 2007

2. Kohn LT, Corrigan J, Donaldson MS. To err is human: building a safer health system. Washington, DC: National Academy Press, 2000

3. Kaufman DW, Kelly JP, Rosenberg L, et al. Recent patterns of medication use in the ambulatory adult population of the United States: the Slone Survey. JAMA 2002;287:337-44.

4. Qato DM, Alexander GC, Conti RM, et al. Use of prescription and over-the-counter medications and dietary supplements among older adults in the United States. JAMA 2008;300:2867-78.

5. Bates DW, Cullen DJ, Laird N, et al. Incidence of adverse drug events and potential adverse drug events. Implications for prevention. ADE Prevention Study Group. JAMA 1995;274:29-34.

6. Bates DW, Boyle DL, Vander Vliet MB, et al. Relationship between medication errors and adverse drug events. J Gen Intern Med 1995;10:199-205.

7. Ackroyd-Stolarz S, Hartnell N, Mackinnon NJ. Demystifying medication safety: making sense of the terminology. Res Social Adm Pharm 2006;2:280-9.

8. Budnitz DS, Shehab N, Kegler SR, et al. Medication use leading to emergency department visits for adverse drug events in older adults. Ann Intern Med 2007:147:755-65

9. Juurlink DN, Mamdani M, Kopp A, et al. Drug-drug interactions among elderly patients hospitalized for drug toxicity. JAMA 2003;289:1652-8.

10. Thomsen LA, Winterstein AG, Sondergaard B, et al. Systematic review of the incidence and characteristics of preventable adverse drug events in ambulatory care. Ann Pharmacother 2007:41:1411-26.

11. Gandhi TK, Weingart SN, Borus J, et al. Adverse drug events in ambulatory care. $N$ Engl J Med 2003;348:1556-64.
12. Gurwitz JH, Field TS, Harrold LR, et al. Incidence and preventability of adverse drug events among older persons in the ambulatory setting. JAMA 2003;289:1107-16.

13. Long CL, Raebel MA, Price DW, et al. Compliance with dosing guidelines in patients with chronic kidney disease. Ann Pharmacother 2004:38:853-8.

14. Mahmood M, Malone DC, Skrepnek GH, et al. Potential drug-drug interactions within Veterans Affairs medical centers. Am J Health Syst Pharm 2007:64:1500-5.

15. Raebel MA, McClure DL, Chan KA, et al. Laboratory evaluation of potassium and creatinine among ambulatory patients prescribed spironolactone: are we monitoring for hyperkalemia? Ann Pharmacother 2007;41:193-200.

16. Mackinnon NJ, Hepler CD. Preventable drug-related morbidity in older adults 1 Indicator development. J Manag Care Pharm 2002;8:365-71.

17. Shrank WH, Polinski JM, Avorn J. Quality indicators for medication use in vulnerable elders. J Am Geriatr Soc 2007;55(Suppl 2):S373-82.

18. National Committee for Quality Assurance. The state of health care quality, 2008 report. http://www.ncqa.org/Portals/0/Newsroom/SOHC/SOHC_08.pdf (Accessed 13 May 2009).

19. Campbell SM, Braspenning J, Hutchinson A, et al. Research methods used in developing and applying quality indicators in primary care. Oual Saf Health Care 2002;11:358-64

20. Shrank WH, Polinski JM, Avorn J. Quality indicators for medication use in vulnerable elders. J Am Geriatr Soc 2007;55(s2):S373-82.

21. Lindblad CI, Hanlon JT, Gross CR, et al. Clinically important drug-disease interactions and their prevalence in older adults. Clin Ther 2006:28:1133-43.

22. Malone DC, Abarca J, Hansten PD, et al. Identification of serious drug-drug interactions: results of the partnership to prevent drug-drug interactions. J Am Pharm Assoc 2004:44:142-51.

23. Morris CJ, Cantrill JA. Preventing drug-related morbidity - the development of quality indicators. J Clin Pharm Ther 2003;28:295-305.

24. Morris CJ, Rodgers S, Hammersley VS, et al. Indicators for preventable drug related morbidity: application in primary care. Qual Saf Health Care 2004;13:181-5.

25. Cockcroft DW, Gault MH. Prediction of creatinine clearance from serum creatinine Nephron 1976;16:31-41.

26. Snow V, Mottur-Pilson C, Gonzales R, for the American College of PhysiciansAmerican Society of Internal Medicine. Principles of appropriate antibiotic use for treatment of nonspecific upper respiratory tract infections in adults. Ann Intern Med 2001; 134:487-9.

27. Zhan C, Sangl J, Bierman AS, et al. Potentially inappropriate medication use in the community-dwelling elderly: findings from the 1996 Medical Expenditure Pane Survey. JAMA 2001;286:2823-9.

28. Hande KR, Noone RM, Stone WJ. Severe allopurinol toxicity. Description and guidelines for prevention in patients with renal insufficiency. Am J Med 1984;76:47-56.

29. Fick DM, Cooper JW, Wade WE, et al. Updating the beers criteria for potentially inappropriate medication use in older adults: results of a US consensus panel of experts. Arch Intern Med 2003:163:2716-24.

30. Manlucu J, Tonelli M, Ray JG, et al. Dose-reducing $\mathrm{H} 2$ receptor antagonists in the presence of low glomerular filtration rate: a systematic review of the evidence. Nephrol Dial Transplant 2005;20:2376-84.

31. Practice Partner DTMS Drug Interaction and Prior Adverse Reaction Reports and MEDI-SPAN Electronic Drug File. Copyright 1995-2005. Wolters Kluwer Health, Inc.

32. Salpeter SR, Greyber E, Pasternak GA, et al. Risk of fatal and nonfatal lactic acidosis with metformin use in type 2 diabetes mellitus: systematic review and meta-analysis. Arch Intern Med 2003;163:2594-602.

33. Nesto RW, Bell D, Bonow RO, et al. Thiazolidinedione use, fluid retention, and congestive heart failure: a consensus statement from the American Heart Association and American Diabetes Association. Diabetes Care 2004;27:256-63.

34. Franse LV, Pahor M, Di Bari M, et al. Hypokalemia associated with diuretic use and cardiovascular events in the systolic hypertension in the elderly program. Hypertension 2000;35:1025-30.

35. Ansell J, Hirsh J, Poller L, et al. The pharmacology and management of the vitamin $\mathrm{K}$ antagonists: the Seventh ACCP Conference on Antithrombotic and Thrombolytic Therapy. Chest 2004;126(3 Suppl):204S-33

36. Crismon ML, Argo TR, Bendele SD, et al. Texas medication algorithm project procedural manual: bipolar disorder algorithms. The Texas Department of State Health Services, 2007. http://www.dshs.state.tx.us/mhprograms/pdf/ TIMABDman2007.pdf (Accessed 29 October 2007).

37. American Diabetes Association, American Psychiatric Association, American Association of Clinical Endocrinologists, et al. Consensus development conference on antipsychotic drugs and obesity and diabetes. Diabetes Care 2004;27:596-601

38. Chiquette $\mathbf{E}$, Amato MG, Bussey HI. Comparison of an anticoagulation clinic with usual medical care: anticoagulation control, patient outcomes, and health care costs. Arch Intern Med 1998;158:1641-7.

39. Ortiz Z, Shea B, Suarez-Almazor M, et al. Folic acid and folinic acid for reducing side effects in patients receiving methotrexate for rheumatoid arthritis. Cochrane Database Syst Rev 1999;(4). CD000951. DOI: 10.1002/14651858.CD000951.

40. Kiefe CI, Weissman NW, Allison JJ, et al. Identifying achievable benchmarks of care: concepts and methodology. Int J Qual Health Care 1998;10:443-7.

41. Weissman NW, Allison JJ, Kiefe $\mathrm{Cl}$, et al. Achievable benchmarks of care: the ABCs of benchmarking. J Eval Clin Pract 1999:5:269-81.

42. Orrico KB. Sources and types of discrepancies between electronic medical records and actual outpatient medication use. J Manag Care Pharm 2008;14:626-31. 